\title{
An Economic Simultaneous Localization and Mapping System for Remote Mobile Robot Using SONAR and an Innovative AI Algorithm
}

\author{
Muhammad Muneeb Saleem
}

\begin{abstract}
This paper proposes an economic and effective approach towards the simultaneous localization and mapping of a mobile rescue robot using a single ultrasonic range finder. The procedure eliminates the complication involved with localizing the robot in a map while creating the map simultaneously, by employing a novel control mechanism. The problem is solved by separating the mapping and localization processes and merging the outputs after specific intervals. The data from sensory devices is processed wirelessly to map the surroundings of the robot on a computer. The innovative $A I$ algorithm extends the map cognitively as subsequent data is acquired. Moreover, a comparison is made between conventional methodologies and the one presented in this paper along with possible enhancements applicable. Using this architecture, the economic cost, computational power and time required for SLAM are significantly reduced. The design was utilized on a mobile rescue robot for the RoboCup competitions.
\end{abstract}

Index Terms-SLAM, SONAR, telerobotics, artificial intelligence

\section{INTRODUCTION}

Simultaneous Localization and Mapping (SLAM) is the problem of building a model leading to a new map or repetitively improving an existing map while at the same time localizing the robot within that map; or the long-term globally referenced position estimation without a priori information[1]. The first step in this procedure involves acquiring raw data from sensors or ranging devices mounted on the robot. This data is processed on a computer to draw the surrounding environment of the robot which mainly consists of the obstacles and walls around the robot. As the robot moves further, more data is obtained from the sensors; either after operator-specified intervals, or in real-time. In any case, the map is extended to include the newly discovered area. Localization involves keeping track of the position of the robot on the generated map simultaneously.

Most robots use vision for simultaneous localization and mapping [2], and rely on particle filtering and other techniques to generate maps [3]. Some robots capable of SLAM use laser range finders (LIDAR)[4], or multidimensional ultrasonic range finders to perceive their surroundings. More innovative approaches are landmark assisted vision [5] and fusion of SONAR with monocular

Manuscript received August 30, 2012; revised October 12, 2012.

Muhammad Muneeb Saleem is with the Faculty of Electronic Engineering, Ghulam Ishaq Khan Institute of Engineering Sciences and Technology, Pakistan. He is now with the University of Texas at Dallas, TX, USA (e-mail: mmuneebsaleem@gmail.com). vision [6]. The former approach has limited practical application as it relies on landmarks, while the latter demands high computational power. Localization is at times done using accelerometers or inertial measurement devices. This approach is an expensive one. Moreover, it involves much complex processing and demands high-end computational power. These requirements are hard to fit specifically in Urban Search and Rescue (USAR) robotics applications.

This paper presents an innovative and economic approach towards SLAM using an inexpensive SONAR sensor, and an innovative and undemanding algorithm for map plotting which makes this design utilizable for consumer robotics applications. In contrast to the aforementioned procedure adopted usually for mapping purposes, a single unidirectional SONAR sensor can be used to sweep through the surroundings of the robot at different points of its route. These data sets can then be remotely processed to create a map which has the ability to repetitively extend itself upon reception of subsequent data sets. Economic localization is accomplished using an optical assembly that detects two-dimensional motion relative to the terrain very similar to that employed in an optical mouse. This system was designed for "SAVIOUR", a rescue robot built for competition in the USAR, Real Rescue Robot League of the RoboCup competitions, and which competed successfully in the RoboCup IranOpen 2010[7].

\section{RoBot DESIGN OVERVIEW}

Sensory devices and actuation circuitry were interfaced with microcontrollers which communicated serially with a $\mathrm{TCP} / \mathrm{IP}$ stack device. The data thus converted to Ethernet protocol was linked with a wireless access point. A video camera was also interfaced with the wireless link to provide live video feed to the base station. The wireless access point sends all acquired data to the remote base computer. The base computer processes the data obtained, and performs map generation and image processing.

Actuation commands are issued back to the remote mobile robot through the same wireless link. Microcontrollers and simple logic circuitry interpret these commands to control the motors and to tap sensor devices for data requisition. This innovative architecture enables the robot to be made compact, robust and lightweight through remote processing. Moreover, the off-board computer makes the processing of complex operations on a heavy-duty processor possible. It allows greater battery lifetime for the robot[8]. Over-rides for autonomous navigation are also more easily realized. In 
addition, the low expense of this design makes it advantageous.

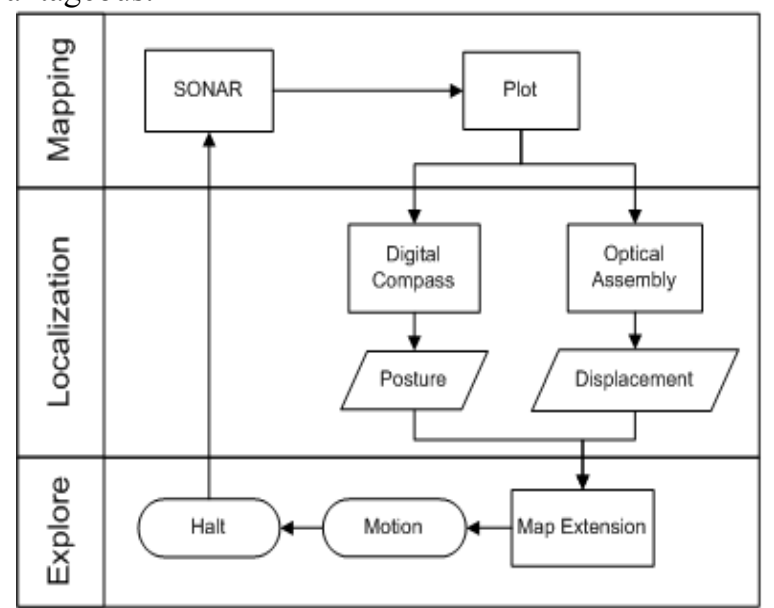

Fig. 1. Flow chart of the basic mechanism of proposed SLAM process.

The aforementioned architecture of the robot was implemented on the "SAVIOUR" rescue robot which made it the most economic robot in the RoboCup Rescue League. The control mechanism worked efficiently.

The system proposed in this paper solves the problems encountered in simultaneous localization and mapping by breaking down the procedure into discrete steps. Mapping and localization are simultaneously performed by dedicating separate equipment for each task. This generates separate sets of data which are managed by the algorithm to extend the map by merging with prior maps or plots. Fig. 1 summarizes the basic architecture of the design in terms of various functional units, data sets and processes.

\section{SONAR INTERFACE}

The core sensor employed for environment detection was a low-cost ultrasonic range finder SRF02. The SRF02 is a single transducer ultrasonic rangefinder in a small footprint PCB. It features both $\mathrm{I} 2 \mathrm{C}$ and serial interfaces. The serial interface is a standard TTL level UART format at 9600 baud, 1 start, 2 stop and no parity bits, and may be connected directly to the serial ports on any microcontroller. Because this ranger uses a single transducer for both transmission and reception, the minimum range is higher than our other dual transducer rangers. The minimum measurement range varies from around 17-18 cm on a warm day down to around 15-16 $\mathrm{cm}$ on a cool day. This minimum range is satisfactory for rescue robotics applications, for example a simulated earthquake hit arena. The SRF02 can measure in both $\mathrm{cm}$ and inches. The manufacturer's beam pattern, showing the sensitivity of the transducer in $\mathrm{dB}$ at different angles is as shown in Fig. 2.

Serial mode of communication was used for the SONAR transducer. It was interfaced with the Atmel 89C51 microcontroller. The transducer is physically mounted on a stepper motor. The stepper motor rotates the ranger in a full circle. This sweep of the ranger at a fixed position at a point on the arena acquires the distances to obstacles and walls around the robot at that point. The stepper motor is stopped momentarily after fixed angles of rotation to acquire data from the sensor. As shown in the sensitivity pattern of the transducer, an effective $30^{\circ}$ interval is selected for optimal data extraction in minimum time. In this manner, the entire $360^{\circ}$ sweep of the surroundings of the robot is completed. The data is sent through the wireless link to the base computer for processing and map generation.

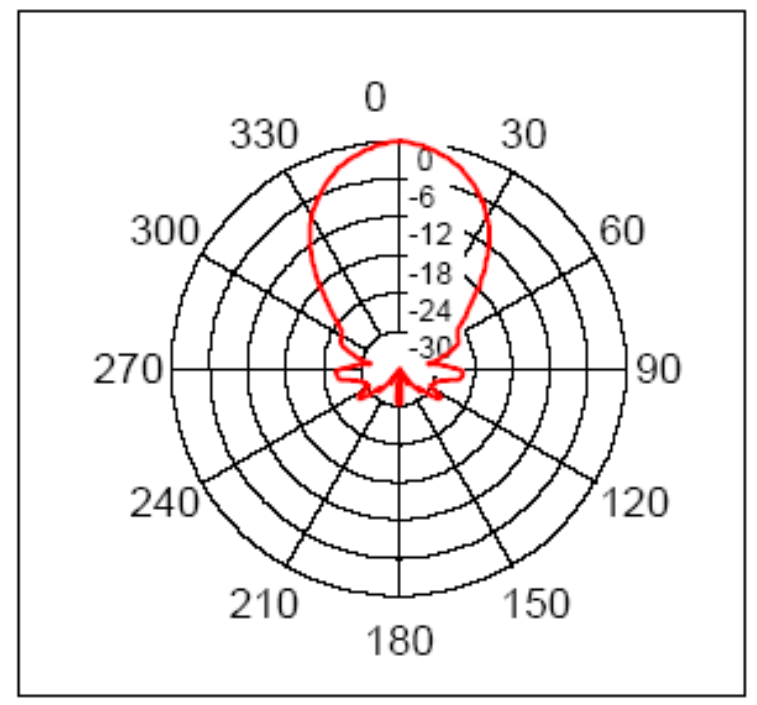

Fig. 2. Sensitivity of the SONAR transducer in $\mathrm{dB}$ at different angles.

The aforementioned procedure is carried out on the robot at relatively different panoramas of the simulated wrecked arena. For advantageous mapping, the panoramas selected have to be adjacent to the preceding panoramas where a transducer sweep has been performed.

The SONAR transducer is highly effective in the USAR robotics applications where very high resolution of the arena is not needed. The scans of SONAR are nevertheless highly effective in finding out accurate distances to and detecting obstacles, slopes, walls etc especially when complimented with the live video feed from the onboard camera. Testing of the transducer resulted in precise measurements as required. This methodology reduced the cost of implementation of SLAM on the robot tremendously.

\section{AI AlgORITHM}

An innovative AI algorithm was essential for effective mapping of all the perceived parameters of the robot's environment visually. The sensor data for mapping along with other data was received by the software based on $\mathrm{C \#}$ on the operator computer. This data was then conveyed to the AI algorithm for recursive mapping and localization.

\section{A. Mapping}

The data from the ultrasonic range finder consisted of radial distances to objects and their corresponding angles. The values of angles are relative to the front or the initial direction of the transducer. As the stepper motor interval was set at $30^{\circ}$, one set of data consisted of 12 values of radial distances to objects around the remote mobile robot. Therefore, one sweep of the SONAR sensor generated one set of data in polar coordinates.

The algorithm converts the data in polar coordinates to Cartesian coordinates and generates a plot of the surroundings. The posture of the differential drive remote 
robot is set according to the reading of the onboard digital compass. The initial orientation of the map canvas is automatically set according to the starting point. This sequential approach reduces the complexity of typical SLAM algorithms and helps in part to solve the problem of simultaneous localization and mapping.

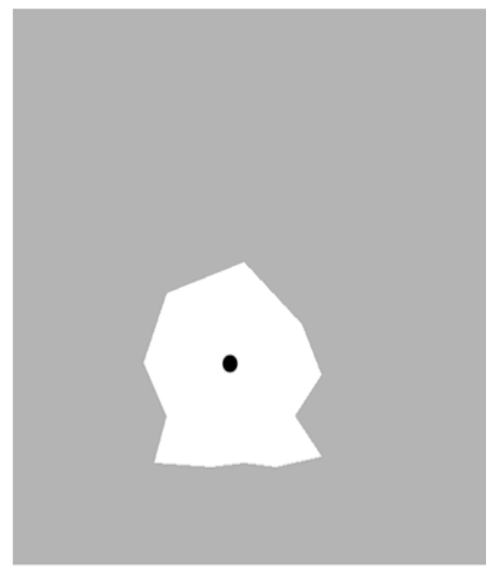

(a) Initial plot

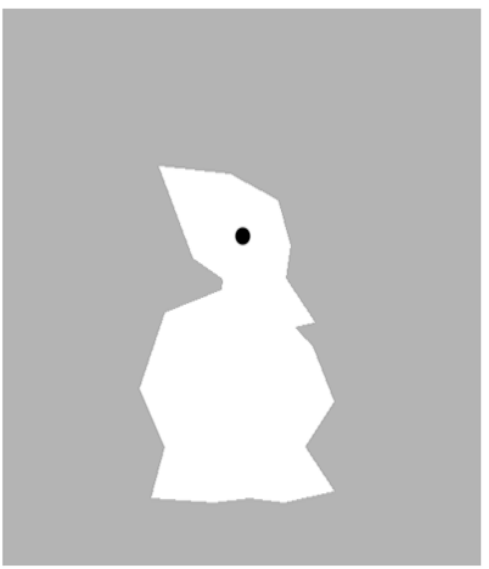

(b) Second plot and extension

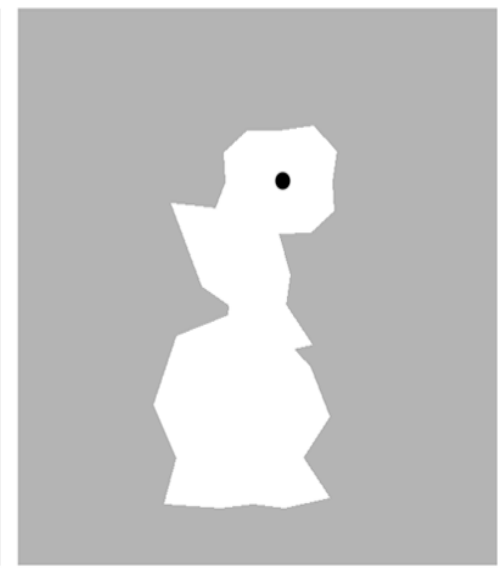

(c) Third plot and the merged resultant map

Fig. 3. Three plots generated from 3 sweeps of SONAR merged to form a part of the map. The black dot shows the location of the robot.

\section{B. Localization}

The data from the digital compass communicated the posture of the differential drive robot while the data from the optical assembly for localization conveyed the exact displacement of the robot. Displacement calculation is accomplished using an optical assembly that detects two-dimensional motion relative to the terrain very similar to that employed in an optical mouse. The algorithm calculates the displacement by counting pixels traversed and then computing them. The AI algorithm calculates the displacement since the last data acquisition sweep; and the digital compass conveys the direction of the robot at that specific point in the arena. These two sets of data solve the problem of localization independent of mapping, and do so effectively and economically.

\section{SLAM}

The AI after preparing the data for mapping and localization intelligently finalizes the map. The first plot generated according to the SONAR data set is sketched on the map canvas in initial posture. Subsequent plots of SONAR transducer data are drawn after simultaneously taking into account the localization data i.e. posture and displacement. These subsequent plots are adjusted according to the posture of the robot (through simple plot rotation) as communicated by the digital compass, and then set on the map canvas at the displacement specified by the optical assembly for localization. Plot images are converted to binary images first to allow binary operations to be carried out on them. When two consecutive plots have an area common to each other as specified by localization, the algorithm logic ORs the images so that no cavity in the arena map is lost. It is highly favorable to make the next sweep at a point from where at least some area of the last sweep lies within the transducer range.

This procedure is carried out recursively automatically as further sweeps are made by the remote mobile robot. The AI algorithm cognitively extends the current map to update the arena geography and the position and posture of the remote rescue robot. The request for transducer sweep can be made automatically after regular intervals as well as manually. The algorithm displays the updated map after each interval of data processing.

The procedure for SLAM suggested in this paper removes the common problem of sequencing the steps involved in SLAM. The problem is the paradox of knowing the map when localizing the robot, while the exact location is needed simultaneously to generate an accurate map in the first place[1]. This problem is resolved by stopping the robot's motion when generating an environment sensor sweep, and then setting the robot in motion while localizing accurately using the optical assembly and digital compass. In short, localization and mapping are successfully carried out in a discrete way rather than simultaneously in real time."

\section{EXPERIMENTAL RESULTS}

Testing of the designed SLAM system is carried out by taking three sweeps of the transducer in Fig. 3. Localization data sets the displacement from the center of first plot to the center of the second plot in two dimensions. Posture data rotates the generated plot in case the robot has changed its direction within the arena. Fig. 3(b) involves linear extension of the initial map. Fig. 3(c) consists of a rotated extension to the main map. The third sweep was at a relative angle of $50^{\circ}$ due to the robot's posture, which has been incorporated into the mapping process by the AI successfully. The black dot shows the position of the robot in the map.

Experimental results show sharp dependence on the resolution of the rotation of stepper motor. An optimal trade-off has to be decided upon when increasing the resolution by decreasing stepper angle, as smaller angles require significantly larger amounts of time to generate SLAM data for processing. Nonetheless, the results are fully compatible with USAR requirements and needs for the remote mobile rescue robot. Dummy victims are identified using the heat, carbon dioxide, sound sensors and the live video feed and then plotted on the maps to allow autonomous navigation towards the victims for further examination and assistance. 


\section{ADVANTAGES}

Besides a few common limitations of the system, the design has major strong points which make it highly favorable for certain applications.

- The design is one of the most cost-efficient and economical compared to the conventional methods of implementation of SLAM.

- Only a single sensor can be used to monitor the surroundings according to this design.

- Robots are made more robust because of the relatively small sizes of the components required.

- Data traffic between the remote robot and the operator station is significantly reduced as data is acquired only when required.

- The AI algorithm is a simple sequential code which saves ample processing power for other uses including image processing tasks etc.

- Power consumption is dramatically reduced allowing much longer durations of robotic explorations.

- The design reduces the architectural complexity of the robot electronics considerably making troubleshooting and handling much easier.

- Problems in synchronization of mapping and localization using a single sensor are solved by using separate sensor sets for both purposes and using only one set at a time.

\section{DEMERITS AND LIMITATIONS}

The SLAM design has several limitations which need further enhancement for rectification. The trade-off between time taken for static scans of the environment and resolution of the plots in terms of sharp edges is the largest factor. Avoidance of real-time scanning brings this disadvantage. Sharp edges have a greater probability of being missed by the SONAR than most laser driven rangers. Moreover, overlapping of consequent plots is an important criterion for the continuity of the paths in the map. Manual scanning of environment can get difficult when this criterion is pursued. SONAR capabilities can be increased by using multiple transducers or employing rangers with low response time. Inaccuracies present in the localization system can be corrected by using an accelerometer.

Maps can be refined by improving feature detection of SONAR transducer[9], [10]. Another method of enhancing results is by the fusion of SONAR with vision[11], or with laser range finders[12], [13].

\section{CONCLUSION}

Use of SONAR transducers for SLAM deployment in remote mobile robots, particularly USAR robots, is highly advantageous in terms of cost, robustness, complexity, computational power and energy conservation. Employment of sequential image based AI algorithm for SLAM significantly reduces required processing power. Most of the decisions are made on the operator base station instead of the onboard processor which further increases economic and processing merits. The disadvantages of the system can be removed simply by extending the hardware capabilities or by using multiple SONAR range finders.

\section{ACKNOWLEDGMENT}

The author would like to acknowledge his team who worked with him on the fabrication of the "SAVIOUR" rescue robot and who was a constant source of inspiration; Dr. M. Junaid Mughal and Muhammad Farooq for their guidance and support, and Ghulam Ishaq Khan Institute of Engineering Sciences and Technology, Pakistan for the opportunity and the facilities.

\section{REFERENCES}

[1] J. J. Leonard and H. F. D. Whyte, "Simultaneous map building and localization for an autonomous mobile robot," in Proceedings International Workshop on Intelligent Robots and Systems '91. Intelligence for Mechanical Systems, vol. 3, Nov. 1991, pp. 1442-1447.

[2] C. Han, Z. Xiang, J. Liu, and E. Wu, "Stereo vision based SLAM in outdoor environments," in Proc. of IEEE International Conference on Robotics and Biomimetics, Dec. 2007, pp. 1653-1658.

[3] D. Marzorati, M. Matteucci, and D. G. Sorrenti, "Particle-based Sensor Modeling for 3D-Vision SLAM," in Proc. of IEEE International Conference on Robotics and Automation, April 2007, pp. 4801-4806.

[4] D. M. Cole and P. M. Newman, "Using laser range data for 3D SLAM in outdoor environments," in Proc. of IEEE International Conference on Robotics and Automation, May 2006, pp. 1556-1563.

[5] X. D. Nguyen, B. J. You, and S. R. Oh, "A Simple Landmark Model for Vision-based Simultaneous Localization and Mapping," in Proc. of International Joint Conference, Oct. 2006, pp. 5016-5021.

[6] H. Y. Liu, L. F. Gao, Y. X. Gai, and S. Fu, "Simultaneous Localization and Mapping for Mobile Robots Using Sonar Range Finder and Monocular Vision," in Proc. of IEEE International Conference on Automation and Logistics, Aug. 2007, pp. 1602-1607.

[7] Islamic Azad University of Qazvin. [Online]. Available: http://www.iranopen2010.ir/Default.aspx?tabid=132\&language=en-G B

[8] P. Rong and M. Pedram, "Extending the lifetime of a network of battery-powered mobile devices by remote processing: a Markovian decision-based approach," Design Automation Conference, 2003. Proceedings, June 2003, pp. 906-911.

[9] J. Choi, S. Ahn, and W. K. Chung, "Robust sonar feature detection for the SLAM of mobile robot," in Proc. of International Conference on Intelligent Robots and Systems, August 2005, pp. 3415-3420.

[10] T. N. Yap and C. R. Shelton, "SLAM in large indoor environments with low-cost, noisy, and sparse sonars," in Proc. of IEEE International Conference on Robotics and Automation, May 2009, pp. 1395-1401.

[11] J. Choi, S. Ahn, M. Choi, and W. K. Chung, "Metric SLAM in Home Environment with Visual Objects and Sonar Features," International Conference on Intelligent Robots and Systems, Oct. 2006, pp. 4048-4053.

[12] A. Diosi and L. Kleeman, "Advanced sonar and laser range finder fusion for simultaneous localization and mapping," in Proceedings of International Conference on Intelligent Robots and Systems, 2004. (IROS 2004), vol. 2, pp. 1854-1859.

[13] A. Diosi, G. Taylor, and L. Kleeman, Robotics and Automation, in Proceedings of the IEEE International Conference on interactive SLAM using Laser and Advanced Sonar, April 2005, pp. 1103-1108.

Muhammad Muneeb Saleem has graduated in electronics engineering from GIK Institute, Pakistan in 2011. He is currently pursuing an MS degree in signal processing and communications at UT Dallas, TX, USA. He has work experience in telecommunications industry. He has published a book titled Biopotential Eye Tracking System (LAP GmbH \& Co., 2011) based on EOG digital signal processing. Research interests include bioelectrical signal processing and pattern recognition. He is a student member of the IEEE 